\title{
Single point insulin sensitivity estimator for predic- ting type 2 diabetes mellitus in obese adolescents
}

\author{
Jaewook Ha', \\ Ye-Rim Oh \\ Eungu Kang, \\ Hyo-Kyoung Nam², \\ Young-Jun Rhie', \\ Kee-Hyoung Lee ${ }^{2}$
}

'Department of Pediatrics, Veterans Health Service, Seoul, Korea

${ }^{2}$ Department of Pediatrics, Korea University College of Medicine, Seoul, Korea
See the commentary on "Single point insulin sensitivity estimator for predicting type 2 diabetes mellitus in obese adolescents" via https://doi.org/10.6065/ apem.2221089edi02.

Received: 26 August, 2021

Revised: 16 September, 2021

Accepted: 15 November, 2021

Address for correspondence: Kee-Hyoung Lee

Department of Pediatrics, Korea University Anam Hospital, Korea University College of Medicine, 73 Inchon-ro, Seongbuk-gu, Seoul 02841, Korea

Email: doclee@korea.ac.kr

https://orcid.org/0000-0002-43199019
Purpose: The prevalence of adolescents with type 2 diabetes mellitus (T2DM) has rapidly increased in Korea over the past few decades with the increase in the number of obese adolescents. The single point insulin sensitivity estimator (SPISE) was recently introduced as a surrogate marker for insulin sensitivity to predict T2DM in adults. We aimed to determine risk factors for T2DM in obese adolescents, including SPISE.

Methods: This retrospective study included 104 adolescents diagnosed with T2DM at Korea University Hospital between January 2010 and December 2020. We compared clinical and biochemical parameters and the SPISE of normoglycemic overweight and obese individuals with those of prediabetic and diabetic adolescents to determine risk factors for T2DM. Receiver operating characteristic analysis was performed with the Youden index to determine the cutoff point of SPISE.

Results: Frequency of fatty liver and family history of T2DM were significantly higher and SPISE level was significantly lower in patients with T2DM than in normoglycemic overweight/obese and prediabetic adolescents $(P<0.01)$. A family history of T2DM, fatty liver, and SPISE value below the cutoff point (4.49) were identified as significant risk factors for T2DM in multiple logistic regression analysis after controlling for age, sex, and body mass index standard deviation score $(P<0.01)$. Conclusion: Family history of T2DM, fatty liver, and low SPISE $(<4.49)$ are risk factors that can independently affect the occurrence of T2DM in obese adolescents. Among these risk factors, SPISE is a promising marker for predicting adolescent T2DM; careful monitoring of these individuals is needed to prevent progression to T2DM.

Keywords: Obese adolescents, Type 2 diabetes mellitus, Single point insulin sensitivity estimator, Fatty liver

\section{Highlights}

- The prevalence of adolescents with type 2 diabetes mellitus (T2DM) has rapidly increased in Korea over the past few decades with the increase in the number of obese adolescents.

. SPISE is a promising marker for predicting adolescent T2DM.

\section{Introduction}

Owing to the spread of western dietary and sedentary behaviors, the incidence of obesity and type 2 diabetes mellitus (T2DM) has increased in Korean adolescents; the percentage of T2DM among all diabetes mellitus adolescents increased four-fold from 5.3\% in 1990 to $21.0 \%$ in 2000 . $^{1)}$

In a 10-year study of type 1 diabetes mellitus (T1DM) and T2DM in adolescents $<15$ years 
of age conducted between 2001 and 2010, T1DM had a higher prevalence than T2DM; however, the increase in prevalence each year was higher for T2DM than T1DM. ${ }^{2)}$

Insulin resistance plays a major role in the development of T2DM. ${ }^{3)}$ Homeostatic model assessment of insulin resistance (HOMA-IR) as an insulin sensitivity index has been used to assess insulin resistance in obese children. ${ }^{4)}$ The single point insulin sensitivity estimator (SPISE) has also been used as a surrogate marker for insulin sensitivity to predict metabolic syndrome and screen for T2DM in adults. ${ }^{5-7)}$ SPISE showed moderate to strong positive correlations with the euglycemic insulin clamp in both adults and juveniles based on an independent study cohort. ${ }^{8}$ However, few studies have investigated the utility of SPISE in adolescents with T2DM.

In this study, we analyzed differences in the proportions and levels of clinical and biochemical indicators, including HOMAIR and SPISE, among normoglycemic overweight or obese adolescents, those with prediabetes, and those with T2DM to identify factors independently associated with T2DM. In particular, we aimed to determine the efficacy of SPISE as a predictor of adolescent T2DM.

\section{Materials and methods}

\section{Subjects}

We retrospectively reviewed the medical records of 104 adolescents ( 60 males and 54 females) aged $<18$ years who were newly diagnosed with T2DM between January 1, 2010, and December 31,2020, at the Korea University Pediatric Clinic. The diagnosis of T2DM was confirmed based on American Diabetes Association guidelines. ${ }^{9)}$ We enrolled 92 normoglycemic overweight/obese adolescents (body mass index $[\mathrm{BMI}] \geq 85$ th percentile for their age and sex, fasting glucose $<100 \mathrm{mg} / \mathrm{dL}$ or 2 -hour glucose $<140 \mathrm{mg} / \mathrm{dL}$ on oral glucose tolerance test [OGTT]) and 59 patients with prediabetes (fasting glucose of $100-125 \mathrm{mg} / \mathrm{dL}$ or 2-hour glucose of $140-199 \mathrm{mg} / \mathrm{dL}$ on OGTT). We recorded age, sex, height, weight, BMI $\left(\mathrm{kg} / \mathrm{m}^{2}\right)$, and the onset of puberty above Tanner stage II. Family history of T2DM was defined as having one or more first- or second-degree relatives diagnosed with T2DM. ${ }^{10)}$ Fatty liver was diagnosed based on the following ultrasound parameters: parenchymal brightness, liver-to-kidney contrast, deep beam attenuation, bright vessel walls, and gallbladder wall definition. ${ }^{11)}$ Acanthosis nigricans was defined as a skin condition characterized by areas of dark, velvety discoloration in body folds and creases. ${ }^{2)}$

\section{Biochemical assessment}

Venous blood samples were taken after an 8-hour fast and analyzed. Fasting glucose, alanine aminotransferase/ aspartate aminotransferase (ALT/AST), total cholesterol (TC), high-density lipoprotein cholesterol (HDL-C), low-density lipoprotein density (LDL-C) cholesterol, and triglyceride (TG) levels were measured by enzyme analysis. Insulin levels were measured by the immuno-radiometry method using ${ }^{125}$ I. The HOMA-IR was calculated based on fasting insulin and glucose using the following equation: HOMA-IR=fasting glucose $(\mathrm{mg} /$ $\mathrm{dL}) \times$ fasting insulin $(\mathrm{mU} / \mathrm{mL}) / 405{ }^{4}{ }^{4}$

The HOMA-IR cutoff point in the pubertal period is 5.22 in boys and 3.82 in girls. ${ }^{4)}$ SPISE was calculated based on HDL and TG levels and BMI using the following equation:

SPISE $=600 \times \mathrm{HDL}-\mathrm{C}^{0.185} /\left(\mathrm{TG}^{0.2} \times \mathrm{BMI}^{1.338}\right)^{8)}$

\section{Statistical analysis}

Data were analyzed using IBM SPSS Statistics ver. 20.0 (IBM Co., Armonk, NY, USA) software. To determine the significance of differences in clinical and biochemical variables between normoglycemic overweight or obese, prediabetic, and T2DM groups, 1-way analysis of variance was used for continuous variables and the chi-square test was used for nominal variables. Receiver operating characteristic (ROC) analysis was performed with the Youden Index to determine the cutoff point of SPISE. Independent variables that showed a significant correlation with T2DM in univariate logistic regression and that did not have a variance inflation factor value of 10 or above were analyzed using multiple logistic regression analysis after adjustment for age, sex, and BMI standard deviation score (SDS) to evaluate risk

Table 1. Clinical variables of adolescents in the normal overweight/obesity, prediabetes, and type 2 diabetes mellitus groups

\begin{tabular}{|c|c|c|c|c|}
\hline Characteristic & $\mathrm{NO}(\mathrm{n}=92)$ & Prediabetes $(n=59)$ & T2DM $(n=104)$ & $P$-value \\
\hline Age at diagnosis (yr) & $14.01 \pm 1.93$ & $13.25 \pm 2.17$ & $14.42 \pm 1.91^{\dagger}$ & $<0.01^{\text {a) }}$ \\
\hline Male sex & $54(58.6)$ & $31(52.5)$ & $60(57.7)$ & $0.742^{b)}$ \\
\hline $\mathrm{BMI}$ at diagnosis $\left(\mathrm{kg} / \mathrm{m}^{2}\right)$ & $27.20 \pm 2.98$ & $27.88 \pm 2.86$ & $28.39 \pm 4.62$ & $0.082^{a)}$ \\
\hline BMI z-score at diagnosis & $2.34 \pm 0.97$ & $2.73 \pm 1.00$ & $2.73 \pm 1.77$ & $0.093^{\mathrm{a})}$ \\
\hline Family history of T2DM & $42(45.7)$ & $28(47.4)$ & $77(74.0)^{*, t}$ & $<0.01^{b)}$ \\
\hline Fatty liver & $26(28.3)$ & $28(47.4)^{*}$ & $76(73.1)^{*, t}$ & $<0.01^{b)}$ \\
\hline Acanthosis nigricans & $61(66.3)$ & $43(72.9)$ & $73(70.2)$ & $0.679^{b)}$ \\
\hline
\end{tabular}

Values are presented as mean \pm standard deviation and number (\%).

$\mathrm{NO}$, normal overweight/obesity; T2DM, type 2 diabetes mellitus; BMI, body mass index.

P-values were calculated by analysis of variance test and ${ }^{\text {b) }}$ chi-square test. For multiple comparisons, Bonferroni post hoc test was performed.

${ }^{*} P<0.05$, compared with the NO group, ${ }^{\dagger} P<0.05$, compared with prediabetes group. 
factors that directly affect T2DM, the binary dependent variable. Continuous data are represented as the mean $( \pm$ standard deviation) and categorical data as the number (\%). A $P$-value less than 0.05 was considered to indicate statistical significance.

\section{Results}

\section{Clinical characteristics of adolescents with T2DM compared to normoglycemic overweight/obese adolescents and prediabetic adolescents}

Average age of T2DM subjects was $14.42 \pm 1.91$ years and their average BMI was $28.39 \pm 4.62 \mathrm{~kg} / \mathrm{m}^{2}$. Mean levels of BMI SDS were similar among groups. Adolescents with T2DM were more likely to have a family history of T2DM among the 3 groups $(45.7 \%, 47.4 \%$ vs. $74.0 \%, P<0.01)$, while the proportion of subjects with a family history of T2DM was similar between adolescents who were normoglycemic overweight/ obese or prediabetic. Adolescents with T2DM had the highest proportion of fatty liver among the three groups $(28.3 \%, 47.4 \%$ vs. $73.0 \%, P<0.01)$, followed by those in the prediabetes and normoglycemic overweight/obese groups. The proportion of acanthosis nigricans was similar among the 3 groups (Table 1).

\section{Biochemical characteristics of adolescents with T2DM compared to those of normoglycemic overweight/ obese adolescents and prediabetic adolescents}

Adolescents with T2DM had the highest levels of AST and ALT among the 3 groups $(46.23 \pm 36.38,70.25 \pm 62.05 \mathrm{mg} / \mathrm{dL}$, $P<0.01$, respectively), followed by those in the prediabetes and normoglycemic overweight/obese groups, respectively. Interestingly, adolescents with T2DM were more likely to have higher levels of TC, TG, and LDL-C (195.35 \pm 42.57 , $164.99 \pm 108.17,125.29 \pm 37.80 \mathrm{mg} / \mathrm{dL}, P<0.01$, respectively) and lower HDL-C levels $(42.15 \pm 8.65, P<0.01)$ among the 3 groups, while levels of TC, TG, LDL-C, and HDL-C were similar between adolescents in the normoglycemic overweight/obese and prediabetes groups.

Adolescents with T2DM had the highest HOMA-IR $(4.95 \pm 2.92, P<0.01)$ among the 3 groups, while HOMA-IR was similar between those in the normoglycemic overweight/obese and prediabetes groups.

SPISE level in patients with T2DM was significantly lower than in patients in the normoglycemic overweight/obese groups, while SPISE level was similar between patients with prediabetes and those with T2DM (Table 2).

\section{Insulin resistance index cutoff point for predicting obese adolescents with T2DM}

More adolescents with T2DM were likely to have a HOMA-IR value above the cutoff point than those in the normoglycemic



Fig. 1. The receiver operating characteristic curve for single point insulin sensitivity estimator in the diagnosis of type 2 diabetes mellitus, which corresponds to the area under the curve ( $95 \%$ confidence interval) of 0.665 (0.5890.741).

Table 2. Biochemical variables of adolescents in the normal overweight/obesity, prediabetes, and type 2 diabetes mellitus groups

\begin{tabular}{|c|c|c|c|c|}
\hline Characteristic & $\mathrm{NO}(\mathrm{n}=92)$ & Prediabetes $(n=59)$ & T2DM $(n=104)$ & $P$-value \\
\hline Glucose at present (mg/dL) & $94.36 \pm 8.76$ & $95.56 \pm 8.57$ & $179.84 \pm 69.82^{*}$ & $<0.01$ \\
\hline $\mathrm{HbA1c}$ at present (\%) & $5.27 \pm 0.26$ & $5.78 \pm 0.22^{*}$ & $9.98 \pm 2.58^{*,+}$ & $<0.01$ \\
\hline AST (mg/dL) & $23.37 \pm 9.44$ & $32.49 \pm 22.20^{*}$ & $46.23 \pm 36.38^{*,+}$ & $<0.01$ \\
\hline $\operatorname{ALT}(\mathrm{mg} / \mathrm{dL})$ & $26.21 \pm 17.98$ & $47.71 \pm 47.60^{*}$ & $70.25 \pm 62.05^{*,+}$ & $<0.01$ \\
\hline Total cholesterol (mg/dL) & $168.07 \pm 30.00$ & $176.76 \pm 29.92$ & $195.35 \pm 42.57^{*, \dagger}$ & $<0.01$ \\
\hline Triglyceride (mg/dL) & $111.40 \pm 55.24$ & $124.54 \pm 68.84$ & $164.99 \pm 108.17^{*},{ }^{+}$ & $<0.01$ \\
\hline HDL-Cholesterol (mg/dL) & $47.82 \pm 11.49$ & $45.84 \pm 9.07$ & $42.15 \pm 8.65^{*, \dagger}$ & $<0.01$ \\
\hline LDL-Cholesterol (mg/dL) & $101.24 \pm 23.51$ & $108.04 \pm 27.14$ & $125.29 \pm 37.80^{*},+$ & $<0.01$ \\
\hline HOMA-IR & $4.95 \pm 2.92$ & $6.26 \pm 3.99$ & $8.42 \pm 6.34^{*,+}$ & $<0.01$ \\
\hline SPISE & $6.01 \pm 1.19$ & $5.67 \pm 1.13$ & $5.33 \pm 1.54^{*}$ & $<0.01$ \\
\hline
\end{tabular}

Values are presented as mean \pm standard deviation.

NO, normal overweight/obesity; T2DM, type 2 diabetes mellitus; HbA1C, glycosylated hemoglobin; HOMA-IR, homeostatic model assessment of insulin resistance; AST, aspartate aminotransferase; ALT, alanine aminotransferase; SPISE-IR, single point insulin sensitivity estimator of insulin resistance.

$P$-values were calculated by analysis of variance. For multiple comparisons, Bonferroni post hoc test was performed.

${ }^{*} P<0.05$, compared with the NO group. ${ }^{\dagger} P<0.05$, compared with the prediabetes group. 
overweight/obese groups ( $63.5 \%$ vs. $44.6 \%, P<0.05)$, while there was no significant difference in number of individuals with a HOMA-IR value above the cutoff point between adolescents in the T2DM and prediabetes groups (63.5\% vs. $59.3 \%, P=0.601)$.

Area under the curve (AUC) was used to determine the diagnostic performance of the SPISE for T2DM. Statistically optimal SPISE cutoff point for T2DM was 4.49 (AUC, 0.665; $95 \%$ confidence interval $[\mathrm{CI}], 0.589-0.741)$, with a sensitivity of $35.6 \%$ and specificity of $94.6 \%$ (Fig. 1 ).

More adolescents with T2DM were likely to have a SPISE values less than 4.49 than those in the prediabetes and normoglycemic overweight/obese groups (35.6\% vs. $11.9 \%$ and $5.4 \%$, respectively, $P<0.01$ ), while the proportion of adolescents with a SPISE value less than 4.49 was similar between the normoglycemic overweight/obese and prediabetes groups.

\section{T2DM risk factor analysis}

In univariate logistic regression analysis, the following risk factors were significantly correlated with T2DM: family history of T2DM (odds ratio [OR], 3.395; 95\% CI, 1.863-6.189; $P<0.01$ ), fatty liver (OR, 6.890; 95\% CI, 3.679-12.904; $P<0.01)$, HOMAIR above the cutoff value (OR, 2.160; 95\% CI, 1.218-3.832; $P<0.01$ ), and SPISE $<4.49$ (OR, 9.609; 95\% CI, 3.582-25.755; $P<0.01)$.

In multiple logistic regression analysis, after adjustment for age, sex, and BMI SDS, family history of T2DM (OR, 3.812; 95\% CI, 1.585-9.166; $P<0.01)$, fatty liver (OR, 4.631; 95\% CI, 1.848 $11.603 ; P<0.01$ ), and SPISE $<4.49$ (OR, 50.307; 95\% CI, 8.053$314.255 ; P<0.01)$ were significantly associated with T2DM. However, a HOMA-IR value above the cutoff point (OR, 1.217; $95 \%$ CI, $0.512-2.891 ; P=0.708)$ was not significantly associated with T2DM in multiple logistic regression analysis (Table 3).

\section{Discussion}

Our study revealed that a family history of T2DM, fatty liver, and SPISE $<4.49$ were independent risk factors for T2DM in obese adolescent. Notably, our results suggest that hyperlipidemia is associated with the development of T2DM.

Adolescent T2DM tends to be present more frequently in those individuals who are obese at the time of puberty and presents with the clinical features of acanthosis nigricans and a family history of T2DM. ${ }^{13-17)}$ In our study, after adjusting for age, sex, and weight, adolescents with T2DM were more likely to have a family history of T2DM than those in the normoglycemic overweight/obese and prediabetes groups. However, the proportion of acanthosis nigricans was similar among the three groups. Some previous studies have reported that insulin resistance plays a role in liver dysfunction such as fatty liver disease, and have found that it contributes to the occurrence of T2DM. ${ }^{18,19)}$

Various methods have been developed to define insulin sensitivity. HOMA-IR is the most widely used indicator of insulin resistance and is based on the feedback loop of glucose and insulin in the postabsorptive state; thus, it is representative of hepatic insulin resistance, but not peripheral insulin sensitivity. ${ }^{20)}$ Factors other than insulin resistance contribute to the increase in glucose levels in T2DM. Therefore, indices based on fasting glucose and insulin levels alone may not accurately track changes in insulin sensitivity. ${ }^{21)}$ Moreover, gender differences in cutoff values should also be considered when evaluating insulin resistance. ${ }^{4)}$ HOMA-IR did not independently affect T2DM in the current study.

Hyperlipidemia has also been used as an indicator of insulin resistance. The liver plays a role in controlling lipid metabolism; therefore, deterioration of hepatic function can lead to hyperlipidemia, thereby resulting in reduced suppression of glucose renewal, which can lead to the development of T2DM. ${ }^{22,23)}$ In a study of hyperlipidemia in T2DM, Chen et al. ${ }^{24)}$

Table 3. Logistic regression analysis for the prediction of type 2 diabetes mellitus (AST/ALT, TG, HDL)

\begin{tabular}{|c|c|c|c|c|}
\hline \multirow{2}{*}{ Variable } & \multicolumn{2}{|c|}{ Unadjusted model } & \multicolumn{2}{|c|}{ Adjusted model } \\
\hline & $\mathrm{OR}(95 \% \mathrm{Cl})$ & $P$-value & OR (95\% Cl) & $P$-value \\
\hline Age & $1.009(0.997-1.022)$ & 0.138 & $1.007(0.994-1.025)$ & 0.425 \\
\hline Male sex & $1.042(0.590-1.841)$ & 0.887 & $0.806(0.339-1.918)$ & 0.626 \\
\hline BMI SDS & $1.210(0.987-1.484)$ & 0.066 & $0.458(0.304-0.691)$ & $<0.01$ \\
\hline Family history of T2DM & 3.395 (1.863-6.189) & $<0.01$ & $3.812(1.585-9.166)$ & $<0.01$ \\
\hline Fatty liver & $6.890(3.679-12.904)$ & $<0.01$ & $4.631(1.848-11.603)$ & $<0.01$ \\
\hline AST & $1.058(1.033-1.083)$ & $<0.01$ & $0.998(0.946-1.053)$ & 0.949 \\
\hline ALT & $1.305(1.021-1.049)$ & $<0.01$ & $1.029(0.998-1.062)$ & 0.069 \\
\hline Triglyceride & $1.010(1.005-1.016)$ & $<0.01$ & $1.002(0.996-1.008)$ & 0.547 \\
\hline HDL-cholesterol & $0.943(0.914-0.974)$ & $<0.01$ & $0.987(0.945-1.030)$ & 0.552 \\
\hline 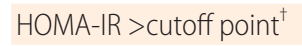 & $2.160(1.218-3.832)$ & $<0.01$ & $1.217(0.512-2.891)$ & 0.657 \\
\hline SPISE $<4.49$ & $9.609(3.582-25.775)$ & $<0.01$ & $50.307(8.053-314.255)$ & $<0.01$ \\
\hline
\end{tabular}

AST, aspartate aminotransferase; ALT, alanine aminotransferase; TG, triglyceride; $\mathrm{HDL}$, high-density lipoprotein; $\mathrm{Cl}$, confidence interval; OR, odds ratio; BMI, body mass index SDS, standard deviation score; T2DM, type 2 diabetes mellitus; HOMA-IR, homeostatic model assessment of insulin resistance; SPISE, single point insulin sensitivity estimator.

${ }^{\dagger}$ The HOMA-IR cutoff point in the pubertal period was 5.22 in boys and 3.82 in girls, respectively. 
revealed that hyperlipidemia is associated with elevated blood glucose levels. In our study, adolescents with T2DM were more likely to have higher levels of TC, TG, LDL-C, and lower HDL-C levels among the three groups, while levels of TC, TG, LDL-C, and HDL-C were similar between those in the normoglycemic overweight/obese and prediabetes groups.

The TG/HDL-C ratio was introduced as a tool to estimate insulin resistance, as circulating lipid measurements are available in routine settings. ${ }^{8)}$ Mathematical modeling was applied to refine the TG/HDL- $\mathrm{C}$ ratio, including $\mathrm{BMI}$, fasting $\mathrm{TG}$, and HDL-cholesterol, and, as a result, the SPISE was invented. SPISE showed a significantly better adjusted ROC than the TG/ HDL-C ratio ${ }^{8)}$ and was used as a surrogate marker for insulin sensitivity to predict metabolic syndrome in adults ${ }^{5)}$ and to screen for T2DM in adults. ${ }^{6,7)}$ Cederholma and Zetheliusb ${ }^{6)}$ revealed that SPISE was a strong predictor of T2DM in all participants, with an OR of $1.62(1.27-2.05 ; P<0.001)$.

To the best of our knowledge, this is the first study to address SPISE in a pediatric population with T2DM in Korea. CorreaBurrows et al. ${ }^{25)}$ reported that the optimal cutoff for metabolic syndrome diagnosis was 5.0 in men and 6.0 in women, and the optimal cutoff for insulin resistance was 5.9 in men and 6.4 in women, respectively. In a European study that investigated SPISE in nondiabetic obese adolescents, a cutoff value for insulin resistance of 6.61 was obtained. ${ }^{8)}$ While there was no significant difference in the proportion of individuals with SPISE $<6.61$ in the normoglycemic overweight/obese and T2DM groups in our study, we found that a SPISE cutoff value of 4.49 predicted T2DM. Adolescents with T2DM were more likely to have a SPISE $<4.49$ among the three groups, and SPISE $<4.49$ independently affected the development of T2DM with an odds ratio of 50.3. SPISE could be applied in clinical studies for risk prediction of T2DM. In our study, the sensitivity of SPISE for predicting adolescent T2DM was only $35.6 \%$, which is insufficient to consider its use as a single screening tool for adolescent T2DM especially given that in a previous study, HOMA-IR was shown to have a sensitivity of $56 \%$ in boys and $77.1 \%$ in girls for predicting T2DM.3) However, the specificity of SPISE in the study was $94.6 \%$, which indicates that T2DM can be ruled out in individuals with a SPISE $>4.49$.

We could not propose a precise cutoff value based on our results due to the retrospective study design and the limited number of patients. Larger prospective studies are needed to determine precise cutoff values.

In conclusion, SPISE, family history of T2DM, and fatty liver are risk factors that can independently predict the occurrence of T2DM. Furthermore, SPISE is a promising marker for predicting adolescent T2DM and should be carefully monitored to prevent progression to T2DM.

\section{Ethical statement}

This study was approved by the Institutional Review Board of Korea University Hospital (approval number: 2020AN0567). The requirement for informed consent was waived due to the retrospective study design.

\section{Notes}

Conflicts of interest: No potential conflict of interest relevant to this article was reported.

Funding: This study received no specific grant from any funding agency in the public, commercial, or not-for-profit sectors.

Data availability: The data that support the findings of this study are available from the corresponding author upon reasonable request.

Author contribution: Conceptualization: JH, KL; Data curation: JH, KL; Formal analysis: JH, KL; Methodology: YO, EK, HN, YR, KL; Project administration: KL; Writing - original draft: JH, KL; Writing - review \& editing: KL

\section{ORCID}

Jaewook Ha: 0000-0001-9063-0820

Ye-Rim Oh: 0000-0001-6087-3119

Eungu Kang: 0000-0001-6544-3599

Hyo-Kyoung Nam: 0000-0003-1512-2062

Young-Jun Rhie: 0000-0002-1250-6469

Kee-Hyoung Lee: 0000-0002-4319-9019

\section{References}

1. Park JM, Yoo EG, Kim DH. Type 2 diabetes mellitus in children. J Korean Pediatr Soc 2002;45:646-53

2. Lee J, Kim Y, Kwak M, Kim S, Kim H, Cheon C, et al. Incidence trends and associated factors of diabetes mellitus in Korean children and adolescents: a retrospective cohort study in Busan and Gyeongnam. Ann Pediatr Endocrinol Metab 2015;20:206-12.

3. Valeria C, Nella P, Cosimo G, Annalisa B, Francesco C. Insulin resistance and type 2 diabetes in children. Ann Pediatr Endocrinol Metab 2020;25:206-12.

4. Kurtolu S, Hatipolu N, Mazioglu M, Kendirci M, Keskin $\mathrm{M}$, Kondolot M. Insulin resistance in obese children and adolescents: HOMA-IR Cut-off levels in the prepubertal and pubertal periods. J Clin Res Pediatr Endocrinol 2010;2:100-6.

5. Dudi P, Goyal B, Saxena V, Rabari K, Mirza AA, Naithani M, et al. Single point insulin sensitivity estimator as an index for insulin sensitivity for metabolic syndrome: a study in North Indian population. J Lab Physicians 2019;11:244-8.

6. Cederholma J,Zetheliusb B. SPISE and other fasting indexes of insulin resistance risks of coronary heart disease or type 2 diabetes. Comparative cross-sectional and longitudinal aspects. Ups J Med Sci 2019;124:265-72.

7. Sagesaka H, Sato Y, Someya Y, Tamura Y, Shimodaira M, Miyakoshi T, et al. Type 2 diabetes: When does it start. J Endocr Soc 2018;2:476-84.

8. Paulmichl K, Hatunic M, Højlund K, Jotic A, Krebs M, Mitrakou A, et al. Modification and validation of the 
Triglyceride-to-HDL cholesterol ratio as a surrogate of insulin sensitivity in White juveniles and adults without diabetes mellitus: the single point insulin sensitivity estimator (SPISE). Clin Chem 2016;62:1211-9.

9. American Diabetes Association. Classification and diagnosis of diabetes: standards of medical care in diabetes-2019. Diabetes Care 2019;42(Suppl 1):S13-28.

10. Danadian K, Jayasekara G, Lewy V, Meza MP, Robertson $\mathrm{R}$, Arslanian SA. Insulin sensitivity in African American children with and without family history of type 2 diabetes. Diabetes Care 1999;22:1325-9.

11. Mahale AR, Prabhu SD, Nachiappan M, Fernandes M, Ullal S. Clinical relevance of reporting fatty liver on ultrasound in asymptomatic patients during routine health checkups. J Int Med Res 2018;46:4447-54.

12. Antonio VS, Carolina A, Helena F. Acanthosis nigricans as a clinical marker of insulin resistance among overweight adolescents. Ann Pediatr Endocrinol Metab 2019;24:99103.

13. Kim J, Lee J. Role of obesity-induced inflammation in the development of insulin resistance and type 2 diabetes: history of the research and remaining questions. Ann Pediatr Endocrinol Metab 2021;26:1-13.

14. John M, Barbara $\mathrm{CH}$. Does obesity cause type 2 diabetes mellitus (T2DM) or is it the opposite. Pediatr Diabetes 2019;20:5-9.

15. Ohlsson C, Bygdell M, Nethander M, Rosengren A, Kindblom JM. BMI change during puberty is an important determinant of adults type 2 diabetes risk in men. J Clin Endocrinol Metab 2019;104:1823-32.

16. Sabin MA, Magnussen CG, Juonala M, Shield JP, Kähönen $\mathrm{M}$, Lehtimäki T, et al. Insulin and BMI as predictors of adult type 2 diabetes mellitus. Pediatrics 2015;135:e144-51.

17. Arslanian SA, Bacha F, Saad R, Gungor N. Family history of type 2 diabetes is associated with decreased insulin sensitivity and an impaired balance between insulin sensitivity and insulin secretion in White youth. Diabetes Care 2005;28:115-9.

18. Bardugo A, Bendor CD, Zucker I, Lutski M, CukiermanYaffe T, Derazne E, et al. Adolescent nonalcoholic fatty liver disease and type 2 diabetes in young adulthood. J Clin Endocrinol Metab 2021;106:e34-44.

19. Newton KP, Hou J, Crimmins NA, Lavine JE, Barlow SE, Xanthakos SA, et al. Prevalence of prediabetes and type 2 diabetes in children with nonalcoholic fatty liver disease. JAMA Pediatr 2016;170:e161971.

20. Hoffman RP, Vicini P, Cobelli C. Pubertal changes in HOMA and QUICKI: relationship to hepatic and peripheral insulin sensitivity. Pediatr Diabetes 2004;5:1225.

21. Pisprasert V, Ingram KH, Lopez-Davila MF, Munoz AJ, Garvey WT. Limitations in the use of indices using glucose and insulin levels to predict insulin sensitivity. Diabetes Care 2013;36:845-53.

22. Lu Y, Wang Y, Zou L, Liang X, Ong CN, Tavintharan S, et al. Serum lipids in association with type 2 diabetes risk and prevalence in a Chinese population. J Clin Endocrinol Metab 2018;103:671-80.

23. Habiba NM, Fulda KG, Basha R, Shah D, Fernando S, Nguyen B, et al. Correlation of lipid profile and risk of developing type 2 diabetes Mellitus in 10-14 year old children. Cell Physiol Biochem 2016;39:1695-704.

24. Chen GY, Li L, Dai F, Li XJ, Xu XX, Fan JG. Prevalence of and risk factors for type 2 diabetes mellitus in hyperlipidemia in China. Med Sci Monit 2015;21:2476-84.

25. Correa-Burrows P, Blanco E, Gahagan S, Burrows R. Validity assessment of the singlepoint insulin sensitivity estimator (SPISE) for diagnosis of cardiometabolic risk in post pubertal Hispanic adolescents. Sci Rep 2020;10:14399. 